\title{
Prevalence of antiretroviral drug resistance among treatment-naive and treated HIV-infected patients in Venezuela
}

\author{
Héctor Rafael Rangel', Domingo José Garzaro', Jaime Rafael Torres², Julio Castro², \\ Jose Antonio Suarez², Laura Naranjo², John Ossenkopp², Nahír Martinez², \\ Resistance Committee Group ${ }^{3}$, Cristina Gutierrez ${ }^{4}$, Flor Helene Pujol'1/+
}

\footnotetext{
'Laboratorio de Virología Molecular, CMBC, Instituto Venezolano de Investigaciones Científicas, Apdo 20632, Caracas 1020-A, Venezuela ${ }^{2}$ Sección de Infectología, Instituto de Medicina Tropical, UCV, Caracas, Venezuela ${ }^{3}$ Resistance Committee Group ${ }^{4}$ Instituto Nacional de Higiene Rafael Rangel, Caracas, Venezuela
}

An in-house, low-cost method was developed to determine the genotypic resistance of immunodeficiency virus type 1 (HIV-1) isolates. All 179 Venezuelan isolates analysed belonged to subtype B. Primary drug resistance mutations were found in $11 \%$ of 63 treatment-naïve patients. The prevalence of resistance in isolates from $116 \mathrm{HIV}$-positive patients under antiretroviral treatment was $47 \%$ to protease inhibitors, $65 \%$ to nucleoside inhibitors and $38 \%$ to non-nucleoside inhibitors, respectively. Around $50 \%$ of patients in the study harboured viruses with highly reduced susceptibility to the three classical types of drugs after only five years from their initial diagnoses.

Key words: HIV - genotypic resistance - diagnostic - Venezuela

Around 1.6 million persons are estimated to be infected with human immunodeficiency virus type 1 (HIV-1) in Latin America, including some 110,000 in Venezuela (UNAIDS/WHO 2007). The most frequently isolated viral type in the region is subtype $\mathrm{B}$, although other subtypes, like subtype $\mathrm{F}$ and $\mathrm{BF}$ recombinant forms, are found in Brazil and the South Cone (Morgado et al. 2002). Highly active antiretroviral therapy (HAART) has been provided free of cost to a total of more than 21,000 patients in Venezuela as of the end of 2007. Genotypic testing by commercial methods adds an additional expense to the high cost of HIV treatment and is performed in Venezuela only for priority cases. Therefore, limited information related to genotypic resistance profiles is available locally. The availability of in-house, affordable genotypic testing offers the opportunity to detect drug resistance mutations even before the start of treatment. Such testing would also allow assessment of the prevalence of primary resistance mutations in the population. The aim of this study was to evaluate genotypic resistance among a representative group of HIV-1-infected Venezuelan patients as well as to assess drug resistance mutation frequencies in a cohort of treated and drug-naïve patients by means of an in-house RT-PCR method.

A total of 179 HIV-1-infected Venezuelan patients were studied, of which 63 patients were naïve and 116 were receiving HAART (most of these later developed virological failure), all predominantly from the national capital Caracas. Signed, informed written consent was

Financial support: FONACIT (G-2005000394), TOTAL Venezuela SA (LOCTI Fund)

+ Corresponding author: fpujol@ivic.ve

Received 4 August 2008

Accepted 3 March 2009 attained in each case. This study was approved by the Instituto Venezolano de Investigaciones Científicas Bioethical Committee. Blood samples were collected between 2004-2007. Information about the most probable mode of transmission and the time of diagnosis of infection was available from 95 and 96 patients, respectively. Adherence information was obtained by a questionnaire designed to determine the number of antiretroviral (ARV) doses missed in the past two weeks, classifying adherence as: $90-100 \%, 1-2$ doses missed; $70-90 \%, 2-3$ doses missed; $50-70 \%, 3-4$ doses missed and $<50 \%$, more than four doses missed.

Viral RNA was extracted from plasma with commercial kits (QIAamp ${ }^{\circledR}$ Viral RNA Kit, QIAGEN, Germany). Around 1,500 nucleotides of the POL gene were amplified by RT and nested PCR, using the following primers: Prot05 (Persaud et al. 2000) and Poll (5'-gttttcagatttttaaatggctcttga- $3^{\prime}$ ) for the first round and Prt15 (Persaud et al. 2000) and Pol 2 (5'-aaatggctcttgataaatttgatatg-3') for the second round. The amplification conditions were: one round of $95^{\circ} \mathrm{C}$ for $2 \mathrm{~min}, 29$ cycles of $90^{\circ} \mathrm{C}$ for $1 \mathrm{~min}$, $55^{\circ} \mathrm{C}$ for $30 \mathrm{sec}$ and $72^{\circ} \mathrm{C}$ for $2 \mathrm{~min}$, with a final extension of $7 \mathrm{~min}$ at $72^{\circ} \mathrm{C}$ and an annealing temperature of $50^{\circ} \mathrm{C}$ for the second round. PCR-purified fragments were sent to Macrogen Sequencing Service (Macrogen, Korea) for sequencing using 5' primers Prt15 and POL2 and 3' extra primers Sp6 (5'-agatatcagtacaatgtgct-3'), A35 (Devereux et al. 2000) and 3Prot2 (Fleury et al. 2003). Sequences were submitted individually to a genotype sequence algorithm (Stanford University HIV Drug Resistance Database, http://hivdb.stanford.edu/index.html).

Overall, 28 HIV-1 plasma samples were tested for genotypic resistance by the in-house method and by a commercial test (Trugene HIV-1/Opengene, Bayer Health Care Laboratories). The sequences obtained by both methods were submitted to the CPR Stanford algo- 
rithm for determination and comparison of drug resistance mutations. Concordant results were obtained in 25 out of 28 sequences. In two of three discordant samples, the mutation M46K or M46I, which generates resistance to protease inhibitors (PIs), was detected by the commercial test. In the other discordant sample, the mutation K219Q was detected only by the commercial test. This mutation did not associate with resistance to additional drugs, as compared to results detected by the in-house test. In total, 158 major drug resistance mutations were detected with the commercial test, while the in-house test detected 152 such mutations ( $96 \%$ correlation). Five out of the six mutations not detected by the in-house test corresponded to quasispecies between the wild type and the mutant amino acid. Of note, analysis of samples by the in-house technique was performed after analysis by the commercial test kit, requiring at least one additional freeze-thawing of plasma, which may have reduced the probability of detecting some of these quasispecies.

All 179 individuals were found to be infected with HIV-1 subtype B, with no evidence of recombination. A total of 63 samples from drug-naïve patients were analysed by the in-house test. Drug-related mutations were found in 24 of these patients (38\%) (Table I). Mutations conferring resistance to at least one ARV drug were found in seven patients (11\%), some of them as quasispecies. In a previous report based on a smaller number of naïve patients from Venezuela (Bouchard et al. 2007), the prevalence of mutations conferring drug resistance $(2 / 20,10 \%)$ was found to be very similar to that observed in the current study $(11 \%)$. The prevalence of drug resistance mutations among viruses infecting naïve Venezuelan patients was higher than those reported in Argentina and Mexico (4.2\% and 2.5\%, respectively) (Dilernia et al. 2007, Viani et al. 2007). In Brazil, primary resistance

\section{TABLE I}

Prevalence of primary drug mutations in HIV-infected treatment naïve patients $(n=63)$

\begin{tabular}{lc}
\hline Mutations & $\begin{array}{c}\text { Patients harboring virus } \\
\mathrm{n}(\%)\end{array}$ \\
\hline None & $39 / 63(62)$ \\
Primary and secondary mutations & $24 / 63(38)$ \\
Mutations conferring drug resistance & $7 / 63(11)$ \\
Primary mutations & \\
PI: M46I & $1(1.6)$ \\
NRTI: K70R & $1(1.6)$ \\
M184V & $2(3.2)^{a}$ \\
T215S & $1(1.6)$ \\
K219E & $1(1.6)$ \\
NNRTI: K103N & $1(1.6)$ \\
G190A & $1(1.6)^{a}$ \\
\hline
\end{tabular}

$a$ : higher occurrence than shown in the HIV data base (CPR Stanford algorithm) for treatment-naïve patients $(p<0.005)$. PI: protease inhibitor; NNRTI: non-nucleoside analog reverse transcriptase inhibitor; NRTI: nucleoside analog reverse transcriptase inhibitor. mutations vary according to region, with low prevalence in Rio de Janeiro (1.4\%) (Varella et al. 2007) and higher prevalence in Sao Paulo (6.5\%) (Gonsalez et al. 2007). The results of primary resistance testing in Venezuela warrant the cost-benefit analysis of a routine, local, primary genotypic resistance surveillance program.

Genotypic resistance was evaluated in a cohort of 116 HIV-positive patients on ARV treatment. The cohort consisted mostly of males (91\%), with an average age of 39.7 years and a predominance of males who have sex with males (MSM; 51/89, 57\%). In the 45 patients for whom adherence to HAART could be assessed reliably, adherence was as follows: more than $90 \%$ adherence in 22 patients (49\%), 70-90\% adherence in $13(29 \%)$ and less than $70 \%$ in $10(22 \%)$. These results suggest that around $50 \%$ of the patients exhibited lower than optimal adherence. The category of resistance found was as follows: $47 \%$ with resistance to PIs, $65 \%$ to nucleoside analog reverse transcriptase inhibitors (NRTIs) and 38\% to nucleoside analog reverse transcriptase inhibitors non-nucleoside analog reverse transcriptase inhibitors (NNRTIs). Of note, $33 \%$ and $18 \%$ of them harboured mutations associated with resistance to both types of reverse transcriptase (RT) inhibitors or to protease and RT inhibitors, respectively. The prevalence of ARV resistance was compared to that reported in the Stanford database ("Mutation prevalence according to subtype and treatment"). A high prevalence of some mutations associated with resistance to NNRTIs, particularly K103N (27\%), was found in this cohort of patients, as compared to the prevalence for this particular mutation reported in the HIV database (18\%). On the other hand, the prevalence of resistance to NRTIs and PIs was generally similar to that reported in the HIV database (Table II). For most of the drugs used by the patients in this study, a significant positive correlation was found between the use of a specific ARV drug and the detection of resistance to that drug. Mutations conferring resistance to a particular drug were more frequently found among patients with a history of receiving that drug. Drug resistance mutations detected were as follows: 42/68 3TC-treated patients vs. $17 / 49$ not treated with $3 \mathrm{TC}(\mathrm{p}=0.001), 43 / 63$ AZT-treated patients vs. $16 / 47$ not treated $(\mathrm{p}<0.001)$, 12/18 RTV-treated patients vs. $12 / 37$ not treated $(\mathrm{p}=$ $0.035), 24 / 38 \mathrm{EFV}$-treated patients vs. 12/37 not treated $(\mathrm{p}<0.001)$ and $26 / 33 \mathrm{ABC}$-treated patients vs. $28 / 84$ not treated $(\mathrm{p}<0.001)$.

The prevalence of mutations conferring resistance to ARV drugs found in treated patients was similar to those described recently in Chile for PIs and NRTIs (46\% and $61 \%$, respectively) and similar to the prevalence reported in Rio de Janeiro, Brazil (70\% to NRTIs, $55 \%$ to NNRTIs and $45 \%$ to PIs) (Bongertz et al. 2007, Rios et al. 2007). This prevalence was also higher than that reported for Venezuela earlier this decade by Delgado et al. (2001). However, resistance to NNRTIs was significantly higher in Chile (84\%) (Rios et al. 2007). The same prevalence of resistance to PIs and NRTIs has been reported in a large study from the USA $(41 \%$ and $71 \%$, respectively), whereas the prevalence of resistance to NNRTIs was significantly lower (25\%) (Richman et 
TABLE II

Prevalence of primary drug mutations in HIV-infected treated patients $(\mathrm{n}=116)$

\begin{tabular}{|c|c|c|c|c|c|c|c|c|c|c|c|c|}
\hline \multicolumn{13}{|c|}{ Mutation associated to resistance to PI: 55/116 (47\%) } \\
\hline D30 & V32 & L33 & M46 & I47 & G48 & I50 & I54 & L76 & V82 & I84 & N88 & L90 \\
\hline $\mathrm{N}(7)$ & $\begin{array}{l}\mathrm{I}(9) \\
\mathrm{T}(1)\end{array}$ & $F(10)$ & $\begin{array}{l}\mathrm{I}(23) \\
L(4)\end{array}$ & $\begin{array}{l}A(2) \\
V(4)\end{array}$ & $V(1)$ & $\mathrm{V}(2)$ & $\begin{array}{c}\mathrm{L}(6) \\
V(25)\end{array}$ & $\mathrm{V}(2)$ & $\begin{array}{c}A(20) \\
\mathrm{C}(1) \\
\mathbf{I}(6) \\
\mathrm{T}(1)\end{array}$ & $\mathrm{V}(16)$ & $\begin{array}{c}\mathrm{D}(10) \\
\mathrm{S}(1)\end{array}$ & $M(27)$ \\
\hline
\end{tabular}

\begin{tabular}{|c|c|c|c|c|c|c|c|c|c|c|c|c|c|c|c|}
\hline \multicolumn{16}{|c|}{ Mutation associated to resistance to NRTI: $75 / 116(65 \%)$} \\
\hline M41 & A62 & D67 & K65 & T69 & K70 & L74 & V75 & F77 & Y115 & F116 & Q151 & M184 & L210 & T215 & K219 \\
\hline L(34) & $\mathrm{V}(6)$ & G(5) & $\mathrm{R}(2)$ & $\mathrm{A}(1)$ & $\mathrm{G}(2)$ & $\mathrm{V}(7)$ & $\mathrm{T}(1)$ & $\mathrm{L}(4)$ & $\mathrm{F}(2)$ & $\mathrm{Y}(4)$ & $\mathbf{M}(9)$ & $\mathrm{V}(49)$ & $\mathrm{F}(1)$ & $\mathrm{C}(1)$ & $\mathbf{E}(\mathbf{1 5})$ \\
\hline \multirow[t]{6}{*}{$\mathrm{K}(1)$} & & $\mathrm{H}(1)$ & & $\mathrm{D}(6)$ & $\mathrm{R}(20)$ & I(7) & $\mathrm{M}(1)$ & & $\mathrm{N}(1)$ & & & $\mathrm{I}(1)$ & $\mathrm{W}(27)$ & $\mathrm{D}(1)$ & $\mathrm{F}(1)$ \\
\hline & & $\mathrm{N}(28)$ & & $\mathrm{N}(5)$ & $\mathrm{E}(1)$ & $\mathrm{L}(1)$ & $\mathrm{I}(3)$ & & & & & & $\mathrm{S}(1)$ & $\mathrm{F}(13)$ & $Q(8)$ \\
\hline & & $\mathrm{I}(1)$ & & $\mathrm{S}(2)$ & $\mathrm{N}(1)$ & & $\mathrm{S}(1)$ & & & & & & & $\mathrm{I}(3)$ & $\mathbf{R}(7)$ \\
\hline & & & & s S_SG (1) & $\mathrm{T}(1)$ & & & & & & & & & $Y(27)$ & $\mathrm{N}(2)$ \\
\hline & & & & & & & & & & & & & & $\mathrm{V}(1)$ & \\
\hline & & & & & & & & & & & & & & $S(3)$ & \\
\hline
\end{tabular}

\begin{tabular}{|c|c|c|c|c|c|c|c|c|c|c|}
\hline \multicolumn{11}{|c|}{ Mutation associated to resistance to NNRTI: 44/116 (38\%) } \\
\hline A98 & L100 & K101 & K103 & V106 & V108 & V179 & Y181 & Y188 & G190 & P225 \\
\hline $\mathrm{G}(5)$ & $\mathbf{I}(\mathbf{6})$ & $\mathrm{P}(2)$ & $\mathrm{N}(30)$ & $\mathbf{I}(\mathbf{6})$ & $\mathrm{I}(5)$ & I(11) & $C(3)$ & $\mathrm{L}(2)$ & $\mathbf{A}(\mathbf{1 1})$ & $\mathrm{H}(3)$ \\
\hline \multirow[t]{4}{*}{$\mathrm{S}(6)$} & & $\mathrm{H}(1)$ & $\mathrm{R}(2)$ & & & $S(1)$ & & & $\mathrm{S}(2)$ & \\
\hline & & $\mathrm{E}(5)$ & S(4) & & & $\mathrm{E}(2)$ & & & $\mathrm{Q}(1)$ & \\
\hline & & $\mathrm{Q}(2)$ & & & & $\mathrm{D}(4)$ & & & & \\
\hline & & $S(1)$ & & & & & & & & \\
\hline
\end{tabular}

the letters in each column correspond to the amino acid found in this position. Numbers under parenthesis correspond to the percent prevalence found in the patients studied. Bold and italic letters indicate higher and lower, respectively, occurrence than the one shown in the HIV data base for treated patients $(p<0.005)$. PI: protease inhibitor; NNRTI: non-nucleoside analog reverse transcriptase inhibitor; NRTI: nucleoside analog reverse transcriptase inhibitor.

al. 2004). A relatively high prevalence $(\mathrm{p}<0.05)$ (Table I) of two PI resistance mutations at codons 47 and 54, which confer decreased susceptibility to several PI drugs, has also been observed previously (Johnson et al. 2006). While the overall prevalence of mutations conferring resistance to NNRTIs found in the current study was not particularly elevated, the observed rate of the NNRTI resistance K103N mutation was rather high $(27 \%)$. This result is similar to a recent report from Brazil (Couto-Fernandez et al. 2005). Moreover, several studies report an increased prevalence of this mutation over time (De Mendoza et al. 2007), which might be related to an increase in viral fitness (Capetti et al. 2005). Alternatively, this mutation might be maintained simply because it has no negative effect on viral fitness.

The number of patients harbouring viruses with susceptibility to only one class of drugs (PI inhibitors, NRTIs and NNRTIs) or to no type of drug was analysed according to the time of diagnosis of infection, for the patients for whom this information was available. In our study, most of the patients requesting genotypic resistance analysis had been diagnosed between five and nine years ago. Moreover, about $50 \%$ of 67 patients already exhibited highly resistant viruses (susceptible to only one class of drugs or to none of these drugs), after as little as five years from the initial diagnosis. Several factors might account for this observation: a relatively late detection of HIV infection, inappropriate use of HAART drugs, lack of availability of important ARV drugs in Venezuela or, finally, poor adherence to treatment. Indeed, a recent study showed that in Venezuela, $40 \%$ of patients receive a diagnosis of HIV-1 infection at a late disease stage (Bonjour et al. 2008). In addition, it must be noted that our cohort study was comprised mainly of patients who were already at a stage of virological failure. Nevertheless, these results emphasise the importance of the rational use of ARV drugs and of adequate counselling for HIV-infected patients in order to maintain adherence to HAART.

\section{REFERENCES}

Bongertz V, Ouverney EP, Fernandez SC, Grinsztejn B, Veloso V, Couto-Fernandez JC, Pilotto JH, Morgado MG 2007. Anti-human immunodeficiency virus type 1 humoral immune response and highly active antiretroviral treatment. Mem Inst Oswaldo Cruz 102: 817-825.

Bonjour MA, Montagne M, Zambrano M, Molina G, Lippuner C, Wadskier FG, Castrillo M, Incani RN, Tami A 2008. Determinants of late disease-stage presentation at diagnosis of HIV infection in Venezuela: a case-case comparison. AIDS Res Ther 5: 6.

Bouchard M, Masquelier B, Moreno M, Deibis L, De Pérez GE, Fleury H, Castro E 2007. HIV type 1 drug resistance among naive patients from Venezuela. AIDS Res Hum Retroviruses 23: 482-485. 
Capetti AF, Gabris AI, Drago L, Vigevani GM 2005. Can a K103N HIV strain stably overcome the wild type in the absence of nonnucleoside reverse transcriptase inhibitor selective pressure? AIDS 19: 633-634.

Couto-Fernandez JC, Silva-de-Jesus C, Veloso VG, Rachid M, Gracie RS, Chequer-Fernandez SL, Oliveira SM, Arakaki-Sanchez D, Chequer PJ, Morgado MG 2005. Human immunodeficiency virus type 1 (HIV-1) genotyping in Rio de Janeiro, Brazil: assessing subtype and drug-resistance associated mutations in HIV-1 infected individuals failing highly active antiretroviral therapy. Mem Inst Oswaldo Cruz 100: 73-78.

De Mendoza C, Garrido C, Corral A, Ramirez-Olivencia G, Jimenez-Nacher I, Zahonero N, Gonzalez-Lahoz J, Soriano V 2007. Changing rates and patterns of drug resistance mutations in antiretroviral-experienced HIV-infected patients. AIDS Res Hum Retroviruses 23: 879-885.

Delgado E, León-Ponte M, Villahermosa ML, Cuevas MT, Deibis L, Echeverría G, Thomson MM, Pérez-Alvarez L, Osmanov S, Nájera R 2001. Analysis of HIV type 1 protease and reverse transcriptase sequences from Venezuela for drug resistance-associated mutations and subtype classification: a UNAIDS study. AIDS Res Hum Retroviruses 17: 753-758.

Devereux HL, Loveday C, Youle M, Sabin CA, Burke A, Johnson M 2000. Substantial correlation between HIV type 1 drug-associated resistance mutations in plasma and peripheral blood mononuclear cells in treatment-experienced patients. AIDS Res Hum Retroviruses 16: 1025-1030.

Dilernia DA, Lourtau L, Gomez AM, Ebenrstejin J, Toibaro JJ, Bautista CT, Marone R, Carobene M, Pampuro S, Gomez-Carrillo M, Losso MH, Salomón H 2007. Drug-resistance surveillance among newly HIV-1 diagnosed individuals in Buenos Aires, Argentina. AIDS 21: 1355-1360.

Fleury H, Recordon-Pinson P, Caumont A, Faure M, Roques P, Plantier JC, Couturier E, Dormont D, Masquelier B, Simon F, Agence Nationale de Recherche sur le SIDA AC11 Laboratory Network 2003. HIV type 1 diversity in France, 1999-2001: molecular characterization of non-B HIV type 1 subtypes and potential impact on susceptibility to antiretroviral drugs. AIDS Res Hum Retroviruses 19: 41-47.
Gonsalez CR, Alcalde R, Nishiya A, Barreto CC, Silva FE, de Almeida A, Mendonça M, Ferreira F, Fernandes SS, Casseb J, Duarte AJ 2007. Drug resistance among chronic HIV-1-infected patients naïve for use of anti-retroviral therapy in São Paulo city. Virus Res 129: 87-90.

Johnson VA, Brun-Vezinet F, Clotet B, Kuritzkes DR, Pillay D, Schapiro JM, Richman DD 2006. Update of the drug resistance mutations in HIV-1: fall 2006. Top HIV Med 14: 125-130.

Morgado MG, Guimarães ML, Galvão-Castro B 2002. HIV-1 polymorphism: a challenge for vaccine development: A review. Mem Inst Oswaldo Cruz 97: 143-150.

Persaud D, Pierson T, Ruff C, Finzi D, Chadwick KR, Margolick JB, Ruff A, Hutton N, Ray S, Siliciano RF 2000. A stable latent reservoir for HIV-1 in resting CD4(+) T lymphocytes in infected children. $J$ Clin Invest 105: 995-1003.

Richman DD, Morton SC, Wrin T, Hellmann N, Berry S, Shapiro MF, Bozzette SA 2004. The prevalence of antiretroviral drug resistance in the United States. AIDS 18: 1393-1401.

Rios M, Delgado E, Perez-Alvarez L, Fernández J, Gálvez P, de Parga EV, Yung V, Thomson MM, Nájera R 2007. Antiretroviral drug resistance and phylogenetic diversity of HIV-1 in Chile. $J$ Med Virol 79: 647-656.

UNAIDS/WHO 2007. Epidemiological fact sheets 2006. AIDS epidemics update, Geneve, p. 1-86.

Varella RB, Ferreira SB, de Castro MB, Zalis MG, Tavares MD 2007. Human immunodeficiency virus type 1 protease and reverse transcriptase mutation patterns among treatment-naive patients in different stages of infection in Rio de Janeiro, Brazil. J Med Virol 79: 1033-1039.

Viani RM, Hsia K, Hubbard P, Ruiz-Calderon J, Lozada R, Alvelais J, Gallardo M, Spector SA 2007. Prevalence of primary HIV-1 drug resistance in pregnant women and in newly diagnosed adults at Tijuana General Hospital, Baja California, Mexico. Int J STD AIDS 18: 235-238. 